(C) 2004 IEEE. Personal use of this material is permitted. Permission from IEEE must be obtained for all other uses, in any current or future media, including reprinting/republishing this material for advertising or promotional purposes, creating new collective works, for resale or redistribution to servers or lists, or reuse of any copyrighted component of this work in other works. 


\title{
Multiresolution Analysis for Reconstruction of Conductivity Profiles in Eddy Current Non- Destructive Evaluation Using Probe Impedance Data
}

\author{
K. R. SHAO, Dezhi CHEN, YouGuang GUO, and J. D. LAVERS
}

\begin{abstract}
This paper presents a wavelet-based multiresolution analysis method for solving the inverse problem in eddy current testing (ECT). Using the probe impedance signals as the measurement data, the reconstruction of conductivity profiles can be performed by a minimization scheme. The method allows to identify the regions where the perturbation may be localized and to retrieve the unknown parameters only in those regions. Some numerical simulation results show the feasibility of the technique.
\end{abstract}

Index Terms-Eddy current testing, inverse problems, multiresolution analysis, probe impedance change.

\section{INTRODUCTION}

$\mathbf{H}$ ddy current non-destructive evaluation of flaws in $\mathrm{E}_{\text {conductive materials has relevance in various industrial }}$ applications. One of the possible solutions of this problem is based on the measurement of the impedance change of a coil generating eddy currents in the conducting material. This technique (ECT) has been extensively studied in the literature [1], [2].

In this paper, we discuss a method for the reconstruction of conductivity profiles from eddy current impedance change data. It is well known [1], [3] that the reconstruction of an unknown conductivity variation from eddy current signal is a nonlinear and ill-posed inverse problem, which can be formalized as the minimization of the mean-square difference between the actually measured and the predicted data derived by solving the related forward problem i.e. the probe-flaw interaction. In this work, we solve the forward problem using an integral equation formulated in terms of an equivalent volume current dipole density at the flaw and containing a dyadic Green's kernel for a half-space conductor.

Wavelet-based multiresolution analysis is used in this paper [4], since the unknown conductivity variations are spatially localized. The method allows to identify regions where the

This work was supported by the National Natural Science Foundation of China (NSFC) under Grant No. 50077009.

K. R. Shao and Dezhi Chen are with the Department of Electrical Engineering, Huazhong University of Science and Technology, Wuhan 430074, China. (e-mail: krshao@mail.hust.edu.cn).

YouGuang Guo is with the Faculty of Engineering, University of Technology Sydney, Australia

J. D. Lavers is with the Department of Electrical and Computer Engineering, University of Toronto, Toronto, Ontario, M5S 3G4, Canada. perturbation may be localized and to reconstruct the unknown coefficients describing perturbation in these regions using high resolution, and low resolution in other regions. This approach also makes that it is possible to analyze adaptively with the smaller number of the variables and save the computation time.

As a numerical example, the conductivity profiles for the steam generator tube ECT benchmark models Step 2 [5] was reconstructed and the parameters of shape and location of the crack were retrieved using the proposed technique. Some numerical results show the main features of the proposed approach.

\section{PROBLEM FORMULATION}

\section{A. Forward Problem}

We start from the expression of eddy current impedance change $\Delta \mathrm{Z}$ due to a flaw. [2]

$$
\Delta Z=-\frac{1}{I^{2}} \int_{\text {flaw }} \frac{\mathbf{P}^{(i)}(\mathbf{r})}{\sigma_{0} v(\mathbf{r})} \cdot \mathbf{P}(\mathbf{r}) d \mathbf{r},
$$

where $\mathbf{P}^{(i)}(\mathbf{r})$ and $\mathbf{P}(\mathbf{r})$ denote the unflawed and the flawed equivalent current dipole densities, which are determined from an integral equation and solved by using the method of moments. $I$ is the driving current of the probe coil.

$$
\text { The flaw function } v(\mathbf{r})=\frac{\sigma(\mathbf{r})-\sigma_{0}}{\sigma_{0}}=\sum_{s=1}^{N} c_{s} \varphi_{s} \text {, }
$$

where $\sigma(\mathbf{r})$ is a spatially varying conductivity and $\sigma_{0}$ is the host conductivity. In this study, we assume that the conductivity perturbation of the flaw will be described by means of the unknown coefficients $C_{S}$ and the shape function $\varphi_{s} . \mathrm{N}$ is the dimension of finite subspace.

The discrete form of (1) is straightforward and can be found in [2]. It is worth to note that here in the integral equation formulation to determine the current dipole density we use the dyadic Green's function of a half-space conductor instead of that of a finite thickness conductor. In fact, by anatomizing the properties of $\mathbf{G}\left(\mathbf{r}, \mathbf{r}^{\prime}\right)$, we have found [6] that for most practical applications in ECT, the former gives fairly good approximations to the latter in cases that the skin depth of eddy current is comparable to the thickness of the conductor. 


\section{B. Nonlinear Optimization}

An optimization approach to this reconstruction seeks the flaw function, which minimizes the mean-square difference between tentative predictions of the probe signals and the measurements. If the agreement is unsatisfactory, then the flaw is updated and a new prediction is made. The process continues through a number of iterations until predictions and observations match to within a reasonable tolerance.

A global "error" $F[v]$ is defined by

$$
F[v]=\sum_{i=1}^{M}\left|\Delta Z_{i}(v)-\Delta Z_{o b s_{i}}\right|^{2},
$$

where $\Delta Z_{o b s}$ is the measured impedance signal, $\mathrm{M}$ is the number of measured data. From (2) and (3), we can see that the inverse problem now amounts to estimate coefficients $c_{1}, \cdots, c_{N}$ of the contrast representation of the flaw function.

The minimization procedure used in this numerical simulation is based on the BFGS algorithm [7], a variable metric method.

\section{MultiResolution ANALYSiS}

The multiresolution analysis (MRA), here briefly recalled, allows to extract those components of a function related to a given spatial scale and position. MRA of $L^{2}(\mathbf{R})$ is defined as a sequence of closed subspace $V_{j}$ of $L^{2}(\mathbf{R}), j \in \mathbf{Z}$, with the following properties:

$$
\begin{aligned}
& V_{j} \subset V_{j+1}, \bigcup_{j \in Z} V_{j}=L^{2}(\mathbf{R}), \bigcap_{j \in Z} V_{j}=\{0\} \\
& f(x) \in V_{j} \Leftrightarrow f(2 x) \in V_{j+1} \\
& f(x) \in V_{0} \Leftrightarrow f(x-k) \in V_{0}
\end{aligned}
$$

Let $V_{0}$ be the linear subspace that contains functions with spatial details up to scale 1 ; from property (4) it follows that $V_{j}$ is the space of functions with spatial details up to scale $2^{-j}$. Let $W_{j}$ be the space defined as the orthogonal complement of $V_{j}$ in $V_{j+1}$; it turns out that $V_{j+1}=V_{j} \oplus W_{j}$. Therefore $W_{j}$ gives the details of $V_{j+1}$ that are lacking in $V_{j}$, i.e. the components at the scale $2^{-(j+1)}$.

For a given multiresolution of $L^{2}(\mathbf{R})$, there exists a scaling function $\Phi(x)$, such that $\left\{2^{j / 2} \Phi\left(2^{j} x-k\right)\right\}_{k \in Z}$ is an orthonormal basis of $V_{j}$, and a wavelet function $\Psi(x)$, such that $\left\{2^{j / 2} \Psi\left(2^{j} x-k\right)\right\}_{k \in Z}$ is an orthonormal basis of $W_{j}$. As a consequence, the following equation is induced:

$$
P_{j+1} f(x)=P_{j} f(x)+\sum_{k=-\infty}^{+\infty} c_{j, k} 2^{\frac{j}{2}} \Psi\left(2^{j} x-k\right),
$$

where $P_{j}$ is the orthogonal projector onto the subspace $V_{j}$. Hence $P_{j} f$ is obtained by filtering out the components related to detail scales finer than $2^{-j}$.

In high dimensional case, tensor product forms is used and the same conclusions above hold. The multiresolution consists of the family $\left\{\hat{V}_{j}\right\}_{j \in Z}$. For two-dimensional case, $\hat{V}_{j+1} \equiv V_{j} \otimes V_{j}$ and $\otimes$ stays the tensor product. It turns out that the scaling function is:

$$
\Phi(x, y)=\Phi(x) \Phi(y),
$$

and the three wavelets are defined as:

$$
\begin{aligned}
& \Psi_{1}(x, y)=\Phi(x) \Psi(y), \\
& \Psi_{2}(x, y)=\Psi(x) \Phi(y), \\
& \Psi_{3}(x, y)=\Psi(x) \Psi(y) .
\end{aligned}
$$

Figure 1 shows the scaling and wavelet functions for 2D Haar system.

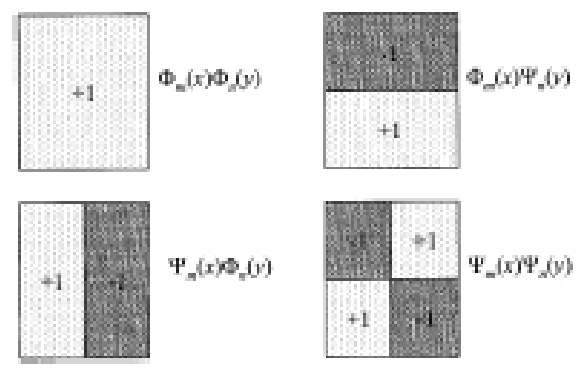

Fig. 1 Prototype two-dimensional Haar scaling and wavelet functions.

\section{NUMERICAL EXAMPLE AND RESUltS}

\section{A. The Example}

Measurement data are from the benchmark models of ECT for steam generator (SG) tube, Step 2 [5]. The coil, crack and conductor parameters are given in Table I. The eddy current skin depth of the conductor is $0.92 \mathrm{~mm}$, while the plate thickness $(1.25 \mathrm{~mm})$ is comparable with. The impedance is measured from the crack center, $\mathrm{y}=0 \mathrm{~mm}$ till $\mathrm{y}=10 \mathrm{~mm}$ at every $1 \mathrm{~mm}$, along crack length direction (Y-axis).

\section{B. Numerical Results}

Using the 11 probe impedance measured data [5], the conductivity profiles of the conductor are reconstructed. Fig. 4 (a) shows the results of analysis and in initial scale on $\hat{V}_{2}$ that is $4 \times 4$ grid. Fig. 4 (b) shows the reanalyzed results on $\hat{V}_{3}$ where $\hat{V}_{3}=\hat{V}_{2} \oplus \hat{W}_{2}$. The reanalyzed results with scale on $\hat{V}_{4}$ are shown in Fig. 4 (c), in which the location and the shape of the flaw are retrieved approximately. The identification of the regions in the center of the conductor around the flaw is analyzed adaptively, and allows avoiding the reconstruction of 
the other regions. Thus the computational time and the computer memory can be greatly saved.

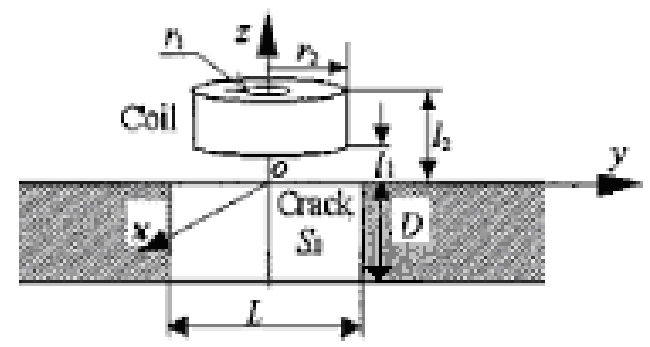

Fig. 2. The pancake type probe coil above a finite-thickness plate with an ideal crack.

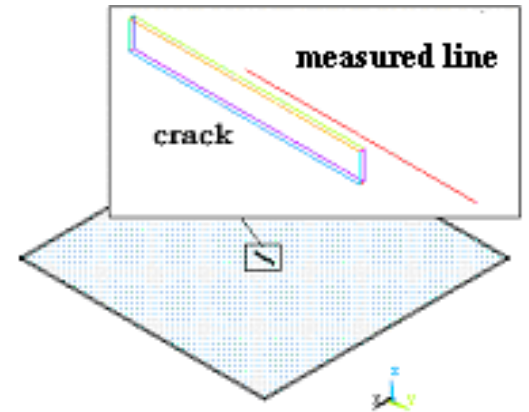

Fig. 3 The measured line

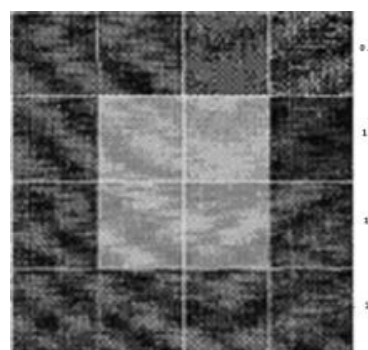

(a)

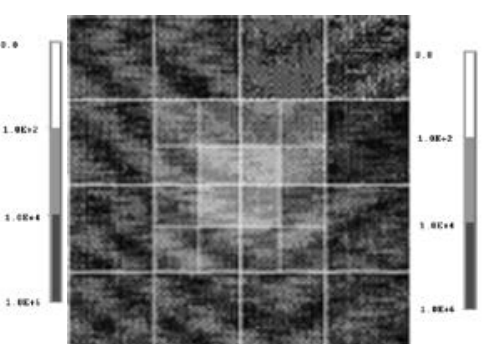

(b)

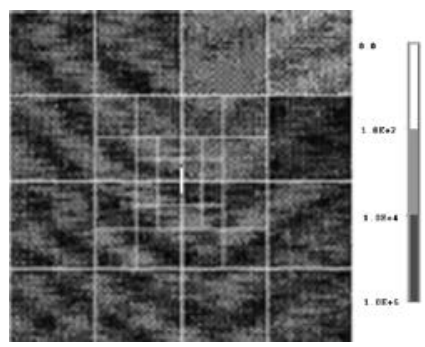

(c)

Fig. 4 Reconstructed conductivity profiles of section of the test domain.

(a) On $\hat{V}_{2}$,

(b) On $\hat{V}_{3}$, where $\hat{V}_{3}=\hat{V}_{2} \oplus \hat{W}_{2}$,

(c) On $\hat{V}_{4}$, where $\hat{V}_{4}=\hat{V}_{3} \oplus \hat{W}_{3}$.

\section{CONCLUSIONS}

A wavelet-based multiresolution analysis method for reconstruction of conductivity profiles of the rectangular conductor model using the probe coil impedance change
TABLE I

COIL, CRACK AND CONDUCTOR PARAMETERS (SEE FIG. 2)

\begin{tabular}{llll}
\hline \hline \multirow{2}{*}{ Coil Parameters } & \multicolumn{3}{c}{ Crack Parameters } \\
\hline inner radius $\mathrm{r}_{1}$ & $0.6 \mathrm{~mm}$ & length $\mathrm{L}$ & $10 \mathrm{~mm}$ \\
outer radius $\mathrm{r}_{2}$ & $1.6 \mathrm{~mm}$ & depth $\mathrm{D}$ & $1.25 \mathrm{~mm}$ \\
liftoff $\mathrm{l}_{1}$ & $0.5 \mathrm{~mm}$ & width & $0.2 \mathrm{~mm}$ \\
\cline { 3 - 4 } top to plate $\mathrm{l}_{2}$ & $1.3 \mathrm{~mm}$ & Conductor & \\
\cline { 3 - 4 } & & Parameters & Inconel 600 \\
\cline { 3 - 4 } driving ac current & $1 / 140 \mathrm{~A}$ & material & $140 \times 140 \times 1.25$ \\
number of turns $\mathrm{N}$ & 140 & size $\left(\mathrm{mm}^{3}\right)$ & $1.0 \times 10^{6} \mathrm{~S} / \mathrm{m}$ \\
\hline frequency & $300 \mathrm{KHz}$ & conductivity $\sigma$ & \\
\hline \hline
\end{tabular}

measurements has been presented. The conventional minimization process is preformed to retrieve the conductivity parameters, which requires a number of iterations for the predictions. In solving the forward problem to obtain the tentative predictions, the integral equation containing a dyadic Green's function for a half-space conductor having analytic formula is employed. Thus the CPU time can be greatly reduced. The multiresolution analysis is a valuable method to identify regions where the components of a function, at a given scale, are located, and to avoid the reconstruction for the other regions.

Some numerical simulation results show the main features and the feasibility of the technique.

\section{REFERENCES}

[1] Stephen J. Norton and John R. Bowler, "Theory of eddy current inversion,” J. Appl. Phys., vol. 73, pp. 501-512, January 1993.

[2] J. R. Bowler, S. A. Jenkins, L. D. Sabbagh, and H. A. Sabbagh, "Eddycurrent probe impedance due to a volumetric flaw," J. Appl. Phys., vol. 70, pp. 1107-1114, August 1991.

[3] J. R. Bowler, S. J. Norton and D. J. Harrison, "Eddy-current interaction with an ideal crack. II. The inverse problem,” J. Appl. Phys., vol. 75, pp. 8138-8144, June 1994.

[4] R. Pierri, G. Rubinacci, A. Tamburrino, S. Ventre and F. Villone, "Non-linear inverse resistivity profiling using wavelets," IEEE Trans. on Magnetics, vol. 34, pp. 2920-2923, September 1998.

[5] T. Takagi, M. Hashimoto, H. Fukutomi, M. Kurokawa, K. Miya and H. Tsuboi, et al, "Benchmark models of eddy current testing for steam generator tube: Experiment and numerical analysis," Int. J. Appl. Electromagn. Mater, vol. 5, pp. 149-162, October 1994.

[6] Dezhi Chen, K. R. Shao and J. D. Lavers, "Very fast numerical analysis of benchmark models of eddy-current testing for steam generator tube," IEEE Trans. on Magnetics, vol. 38, pp. 2355-2357, September 2002.

[7] W. H. Press, S. A. Teukoolsky, W. T. Vetterling, and B. P. Flannery, Numerical Recipes in C, Cambridge University Press 